\title{
Host immune response in returning travellers infected with malaria
}

Gregory MacMullin 1,2, Ronald Mackenzie ${ }^{1,3}$, Rachel Lau', Julie Khang ${ }^{4}$, Haibo Zhang ${ }^{3,4}$, Nimerta Rajwans ${ }^{5}$, W Conrad Liles ${ }^{3,5}$ and Dylan R Pillai ${ }^{6^{*}}$

\begin{abstract}
Background: Clinical observations suggest that Canadian-born (CB) travellers are prone to more severe malaria, characterized by higher parasite density in the blood, and severe symptoms, such as cerebral malaria and renal failure, than foreign-born travellers (FB) from areas of malaria endemicity. It was hypothesized that host cytokine and chemokine responses differ significantly in CB versus FB patients returning with malaria, contributing to the courses of severity. A more detailed understanding of the profiles of cytokines, chemokines, and endothelial activation may be useful in developing biomarkers and novel therapeutic approaches for malaria.
\end{abstract}

Materials and methods: The patient population for the study $(n=186)$ was comprised of travellers returning to Toronto, Canada between 2007 and 2011. The patient blood samples' cytokine, chemokine and angiopoietin concentrations were determined using cytokine multiplex assays, and ELISA assays.

Results: Significantly higher plasma cytokine levels of IL-12 (p40) were observed in CB compared to FB travellers, while epidermal growth factor (EGF) was observed to be higher in FB than CB travellers. Older travellers (55 years old or greater) with Plasmodium vivax infections had significantly higher mean cytokine levels for IL-6 and macrophage colony-stimulating factor (M-CSF) than other adults with $P$. vivax (ages 18-55). Patients with $P$. vivax infections had significantly higher mean cytokine levels for monocyte chemotactic protein-1 (MCP-1), and M-CSF than patients with Plasmodium falciparum. Angiopoietin 2 (Ang-2) was higher for patients infected with $P$. falciparum than P. vivax, especially when comparing just the FB groups. IL-12 (p40) was higher in FB patients with $P$. vivax compared to $P$. falciparum. II-12 (p40) was also higher in patients infected with $P$. vivax than those infected with Plasmodium ovale. For patients travelling to West Africa, IFN- $\gamma$ and IL-6 was lower than for patients who were in other regions of Africa.

Conclusion: Significantly higher levels of IL-12 (p40) and lower levels of EGF in CB travellers may serve as useful prognostic markers of disease severity and help guide clinical management upon return. IL-6 and M-CSF in older adults and MCP-1, IL-12 (p40) and M-CSF for P. vivax infected patients may also prove useful in understanding age-associated and species-specific host immune responses, as could the species-specific differences in Ang- 2 . Regional differences in host immune response to malaria infection within the same species may speak to unique strains circulating in parts of West Africa.

Keywords: Malaria, Cytokines, Chemokines, Angiopoietins, Immunology, Severity

\footnotetext{
* Correspondence: drpillai@ucalgary.ca

${ }^{6}$ Current address: Departments of Pathology \& Laboratory Medicine,

Medicine, and Microbiology \& Infectious Diseases, The University of Calgary,

Diagnostic \& Scientific Centre, Room 1W-416, 9-3535 Research Road NW,

Calgary, AB T2L 2K8, Canada

Full list of author information is available at the end of the article
} 


\section{Background}

Almost half the global population is at risk of contracting malaria, with 300-500 million new clinical cases arising each year that result in between one and three million deaths $[1,2]$. In countries where malaria is non-endemic, the parasite can still have a significant impact on the population and its healthcare system. When travellers visit malaria endemic regions, especially when visiting friends and relatives (VFR), there is a significant risk of the disease being imported into a non-endemic country [3,4]. Each year, approximately 10,000-30,000 travellers from industrialized nations are expected to contract malaria, and the number is continuing to increase [5]. Toronto, Ontario has a population of 2.48 million people $(5.5$ million in the Greater Toronto Area), with half of the inhabitants having been born outside Canada [6]. In addition to the large population, over 16 million people visit the city each year [7]. The substantial immigrant population and associated factors create significant potential for malaria cases to occur in the region, despite it being non-endemic.

Severe malaria is defined by the WHO as a situation in which a patient suffering from asexual parasitaemia, with no other obvious causes of symptoms, has one or more clinical or laboratory features that include but are not limited to hyperparasitaemia, impaired consciousness or unrousable coma, renal impairment, and prostration [8,9]. Severe malaria is most often seen in Plasmodium falciparum-infected patients, but has been seen, defined under the same criteria, in Plasmodium vivax-infected patients [9]. Clinical observations of Canadian-born (CB) travellers suggest they are prone to more severe malaria compared to foreign-born (FB) travellers[10]. It was hypothesized that cytokine, chemokine, and endothelial activation responses would differ significantly between $\mathrm{CB}$ and $\mathrm{FB}$ travelling patients returning with malaria, as more severe cases of malaria often have characteristically different immune response profiles [11-13]. To address this hypothesis, cytokine, chemokine, and expression of the endothelial activation marker angiopoietin-1/2 (Ang-1/2) was quantified based on $\mathrm{CB}$ and $\mathrm{FB}$ status, gender, age group, country of travel, and species of Plasmodium ( $P$. vivax, $P$. falciparum, Plasmodium malariae and Plasmodium ovale) infection. The resulting data was analysed to identify immune factors associated with disease severity, and other patient sample characteristics.

\section{Methods}

\section{Ethics approval}

The study acquired ethics approval from the Toronto Academic Health Sciences Network Research Ethics Board (REB\#10-0094-E).

\section{Study population and patient inclusion criteria}

The malaria specimen database at Public Health Ontario Laboratories (PHOL) comprises patient blood samples received for analysis between 2006 and 2011 coupled to basic patient information. Samples from patients suspected to have malaria were obtained via venipuncture by physicians from hospitals and clinics throughout the Greater Toronto Area and transported to PHOL in EDTA vacutainer (BD) tubes for processing in the clinical parasitology department. Following sample analysis using Giemsastained thin and thick smear microscopy, in vitro immunochromatographic assays (BinaxNow, Inverness Medical), and quantitative PCR as required, samples positive for malaria were transferred to molecular research for storage and further analysis.

Only samples with complete demographic information, defined as patient age, gender, parasitaemia, country of birth and species of infection, were selected for the study. The sample set was stratified into patients born in Canada, and those who recently immigrated to Canada from malaria endemic regions. The samples from $\mathrm{CB}$ patients were considered to be more likely to have severe malaria, as defined by WHO guidelines and the RIFLE criteria for acute renal failure $[8,9]$. An approximately even number of males and females were selected, with a distribution of ages and parasitaemia. Samples were selected from patients infected with each of the four malaria parasites, generating four distinct sample sets to study. A total of 186 patient samples were analysed in this study. Detailed sample information is depicted in Table 1.

\section{Sample processing and storage}

After homogenization through repeated inversions, specimens delivered to Public Health Ontario, which were positive for $P$. vivax, $P$. ovale, and $P$. malariae were immediately transferred into $110 \mu \mathrm{L}$ whole-blood aliquots. The aliquots were stored in $0.5 \mathrm{~mL}$ screw top tubes at $-80^{\circ} \mathrm{C}$. Specimens positive for $P$. falciparum were first separated into two approximately equal aliquots after homogenizing. One aliquot was further separated into cellular and plasma fractions via centrifugation at 2,000 rpm for $5 \mathrm{~min}$. The plasma fraction and the remaining whole blood aliquot were separated into $110 \mu \mathrm{L}$ volumes and stored at $-80^{\circ} \mathrm{C}$ in the same manner as the other Plasmodium spp.

Patient samples arrived at PHOL along with specimen data sheets filled out by their attending doctor specifying age, gender, travel history, and other personal data. Clinical parasitology test results confirmed species, immunochromatography test results, parasitaemia (parasites $/ 1,000$ cells counted), and microscopy as well as PCR observations. The data was de-linked from the patients' names and other personal identifiers, and the remaining details were added 
Table 1 Detailed sample information, broken down by species of infection, Canadian-born or foreign-born status, sex and gender

\begin{tabular}{|c|c|c|c|c|}
\hline & P. falciparum & P. vivax & P. ovale & P. malariae \\
\hline & $n=103$ & $n=60$ & $n=14$ & $\mathrm{n}=9$ \\
\hline Canadian-born & 54 & 12 & 1 & 2 \\
\hline \multicolumn{5}{|l|}{ Sex } \\
\hline Male & 34 & 9 & 0 & 0 \\
\hline Female & 20 & 3 & 1 & 2 \\
\hline \multicolumn{5}{|l|}{ Age } \\
\hline 0-18 & 6 & 0 & 0 & 0 \\
\hline 19-55 & 38 & 9 & 1 & 2 \\
\hline $55+$ & 10 & 3 & 0 & 0 \\
\hline $\begin{array}{l}\text { Foreign-born } \\
\text { (Recent immigrant) }\end{array}$ & 49 & 48 & 13 & 7 \\
\hline \multicolumn{5}{|l|}{ Sex } \\
\hline Male & 18 & 26 & 9 & 5 \\
\hline Female & 31 & 22 & 4 & 2 \\
\hline \multicolumn{5}{|l|}{ Age } \\
\hline $0-18$ & 12 & 8 & 1 & 0 \\
\hline 19-55 & 30 & 26 & 11 & 6 \\
\hline $55+$ & 7 & 15 & 1 & 1 \\
\hline
\end{tabular}

Table 1 - Sample Set Information.

to a computer database, with an original copy of the specimen data sheet being placed under lock and key for reference. Follow-ups with the patients' physicians by the medical microbiologist occasionally provided more specific patient information.

Samples selected for use in the study required additional processing prior to being used in the assays. All $P$. falciparum samples designated for use in the experiment were from plasma aliquots. The aliquots were removed from $-80^{\circ} \mathrm{C}$ storage, and thawed on ice until they were ready for aspiration. The samples were then separated into two $55 \mu \mathrm{L}$ aliquots and placed back in $-80^{\circ} \mathrm{C}$ storage until required for assays.

Whole-blood samples of $P$. vivax, $P$. ovale, and $P$. malariae were removed from $-80^{\circ} \mathrm{C}$ storage and after thawing on ice, treated with lysis buffer in order to isolate the plasma fraction. This step was required because repeated freeze/thawing of the blood at $-80^{\circ} \mathrm{C}$ leads to some cell lysis, releasing haemoglobin into the plasma fraction and inhibiting proper separation by pipetting and visible plasma fraction. To ensure the samples contained no intact erythrocytes, they were incubated in ACK lysing buffer (Invitrogen) at a ratio of 10:1 sample to buffer volume for $3 \mathrm{~min}$ at room temperature. This step was followed by centrifugation of the samples at $4^{\circ}$ $\mathrm{C}$ and 2,000 rpm for $5 \mathrm{~min}$. Following this, the supernatant was removed and stored in $55 \mu \mathrm{L}$ aliquots back at $-80^{\circ} \mathrm{C}$ until required for assays.
The prepared samples were transported to collaborators on dry ice and immediately placed at $-80^{\circ} \mathrm{C}$ upon arrival.

\section{Cytokine multiplex assay}

Based on prior studies that established a link either in vitro or in vivo with malaria infection, 20 cytokines were selected to evaluate in this study [11-19]. These included; GM-CSF, IFN- $\gamma$, IL-1b, IL-2, IL-4, IL-6, IL-8, IL-10, TNF, IL-12(p70), MCP-1, IL-5, TGF- $\alpha$, TGF- $\beta$, IL-12(p40), IL-13, IL-17- $\alpha$, EGF, M-CSF, and IFN- $\beta$. Cytokine quantification was conducted using the Procarta ${ }^{\circledR}$ Cytokine Assay Kit (Affymetrix, Santa Clara, CA, USA) [20]. The final concentrations of cytokine and chemokines are reported in $\mathrm{pg} / \mathrm{mL}$.

\section{Angiopoietin-1/2 ELISA assay}

Based on prior studies establishing a link between severe malaria and angiopoietins, Ang-1 and Ang-2 were also evaluated $[12,19]$.

To do this, 96-well plates were coated with $50 \mu \mathrm{l}$ of capture antibody (R\&D; Ang-1: cat. DY923, Ang-2: cat. DY623) per well and left to incubate overnight at room temperature. Plates were then washed seven times with 0.05\% Tween-20 in PBS pH 7.2-7.4, and blotted on paper towel. Two hundred $\mu \mathrm{l}$ of reagent diluent (PBS pH $7.2-7.4 / 0.1 \%$ BSA mixture) was then applied to each well and left to incubate for $2 \mathrm{~h}$. Afterwards, the diluent was drained and $100 \mu \mathrm{l}$ of standard solution (R\&D; part 940685) diluted in reagent diluent was applied to standard wells at the top of the standard lanes. The remaining wells in the standards lane were filled with standards serially diluted, so that each well contained half the concentration of standards compared to the well above it, with the last lane containing only reagent diluent. The remaining wells were filled with $50 \mu \mathrm{l}$ of sample diluted at a ratio of 1:5. The plates were then left to incubate at $4^{\circ} \mathrm{C}$ overnight.

Ninety-six-well plates were then washed seven times with $0.05 \%$ Tween-20 in PBS pH 7.2-7.4, and blotted on paper towel. Fifty $\mu$ l of detection antibody (R\&D: part 840684) reconstituted in reagent diluent was then applied to each well and left to incubate for $2 \mathrm{~h}$ at room temperature. Plates were then washed seven times with 0.05\% Tween-20 in PBS pH 7.2-7.4, and then blotted on paper towel. Fifty $\mu \mathrm{l}$ of extravidin alkaline phosphatase (Sigma; cat. E2636) that was diluted 1:1,000 in reagent diluent was applied to each well and left to incubate for $45 \mathrm{~min}$ at room temperature. Plates were washed another seven times with $0.05 \%$ Tween-20 in PBS pH 7.27.4 , then rinsed with distilled water and blotted on paper towel. One hundred $\mu$ l of PNPP Sigma fast (Sigma; cat. $\mathrm{N} 2770$ ) was then applied to each well and the plate was 
left to develop in the dark until ready. The top standards well should be between two and three at an absorbance of $405 \mathrm{~nm}$. The optical density was then determined at a wavelength between $405-570 \mathrm{~nm}$, and a final cytokine concentration was determined in $\mathrm{pg} / \mathrm{mL}$.

\section{Statistical analysis}

Cytokine and Ang-1/2 data were broken down into various categories for analysis. The primary focus was on significant differences between $\mathrm{FB}$ and $\mathrm{CB}$ data using an unpaired student's $t$-test. Additional comparisons were made for the data set based on patient travel history, age, gender, and species of infection. Samples for which cytokine or Ang-1/2 testing did not yield data due to technical difficulties were excluded. Each comparison was completed using the student's unpaired $t$-test to assess significant differences between the related data sets defined by a $p$ value $<0.05$ (Additional file 1: Table S1). The values are reported as mean $\pm \mathrm{SD}$. Data analysis was completed using GraphPad Prism (La Jolla, CA, USA) graphing software.

\section{Results}

\section{Immune response by birth status}

The cytokine levels indicated a variety of significant differences when the data was analysed by $\mathrm{CB}$ vs FB, gender, travel history, and ages grouped by $0-18,19-55$, and 55+ (Table 2). When divided into $\mathrm{CB}$ and $\mathrm{FB}$ groups, a significantly higher level of IL-12 (p40) was found in $\mathrm{CB}$ travellers than in FB travellers $(p<0.01)$ (Figure 1). When the P. falciparum-infected patients were examined alone, the $\mathrm{CB}$ travellers also had a significantly higher level of IL-12 (p40) compared to FB travellers $(p=<0.01)$. A significantly higher level of EGF $(p=0.0315)$ was found to exist in $\mathrm{FB}$ compared to $\mathrm{CB}$ travellers (Figure 1).

\section{Immune response by age and species}

When grouped by ages, $P$. vivax infections indicated a tendency for older adults to have a more robust immune response than younger adults for two different cytokines. Those individuals aged 55 and over demonstrated a higher level of IL-6 $(p=0.0366)$, and M-CSF $(p=0.0433)$, than those aged 19-55 (Figure 2).

Cytokine responses for individuals infected with $P$. ovale, $P$. malariae, P. vivax, and P. falciparum were compared and significant differences in the mean level of several cytokines were found when looking at all patient data, comparisons of $\mathrm{CB}$ patients only, and comparisons $\mathrm{FB}$ patients only. Patients infected with $P$. vivax had higher levels of MCP-1 $(p=<0.0001)$ and M-CSF $(p=<0.0001)$ than patients infected with $P$. falciparum. Patients infected with $P$. vivax had higher levels of IL-12 (p40) $(p=0.0478)$ than patients infected with $P$. ovale. $\mathrm{CB}$ patients infected with $P$. vivax also had higher levels of MCP-1 $(p=<0.0001)$, and M-CSF ( $p=0.0367)$, when compared to CB patients infected with $P$. falciparum. FB patients infected with $P$. vivax had higher levels for MCP-1 $(p=0.0020)$, M-CSF $(p=<0.0001)$, and also IL-12(p40) $(p=0.0467)$, compared to FB patients infected with P. falciparum (Figure 3).

\section{Immune response by region of travel}

Levels of IFN- $\gamma(p=0.0414)$ and IL-6 $(p=0.0426)$ were significantly lower in patients who travelled to West Africa compared to those who had travelled to other regions in Africa (Figure 4).

\section{Endothelium activation based on angiopoietin levels}

The Ang- $1 / 2$ concentrations showed no significant difference between FB and CB patient groups. Additional analysis by gender, travel history, and ages grouped by $0-18$, 19-55, and 55+, showed no significant differences for
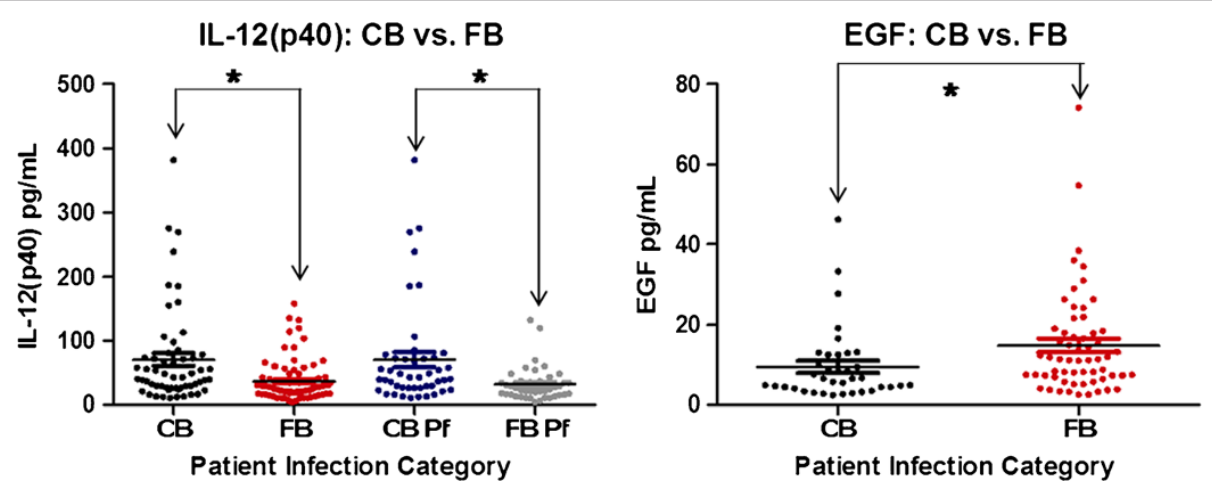

Figure 1 Canadian-born (CB) and Foreign-born (FB) significant differences. Significant differences for IL-12 (p40) and EGF were observed between CB and FB travellers. Each coloured circle represents a single patient, with a horizontal line through each data set indicating the mean concentration. Data sets are compared to one another by arrows spanning between them along with an asterix. See Table 2 for $p$ values. 
IL-6: Pv Age Breakdown

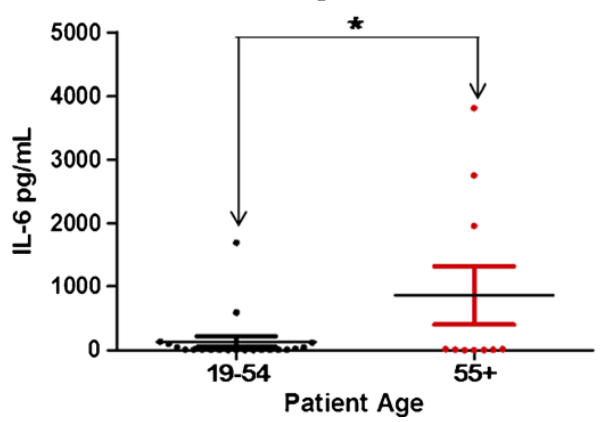

M-CSF: Pv Age Breakdown

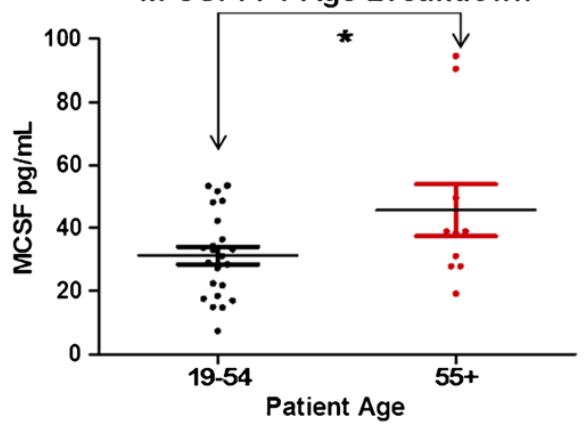

Figure 2 Age-based significant differences. Significant differences for IL-6 and M-CSF were observed between 19-54 year olds and those 55 and older who were infected with Plasmodium vivax. Each coloured circle represents a single patient, with a horizontal line through each data set indicating the mean concentration. Data sets are compared to one another by arrows spanning between them along with an asterix. See Table 2 for $p$ values.

either Ang-1 or Ang-2 levels in the patient plasma samples. A significantly higher level of Ang-2 $(p<0.01)$ was observed in patients infected with P. falciparum, compared to those infected with $P$. vivax. The Ang-2 levels were also significantly different $(p=0.0218)$ between the FB patients infected with each species, with a higher mean present for FB P. falciparum than for FB P. vivax (Figure 3).

\section{Discussion}

This study was conducted on returning travellers who contracted malaria in order to evaluate the cytokine, chemokine, and endothelial activation response in human infection. We hypothesized that in cities like Toronto travellers infected with malaria who are $\mathrm{CB}$ will elaborate a different immune response to those who are FB. This response was

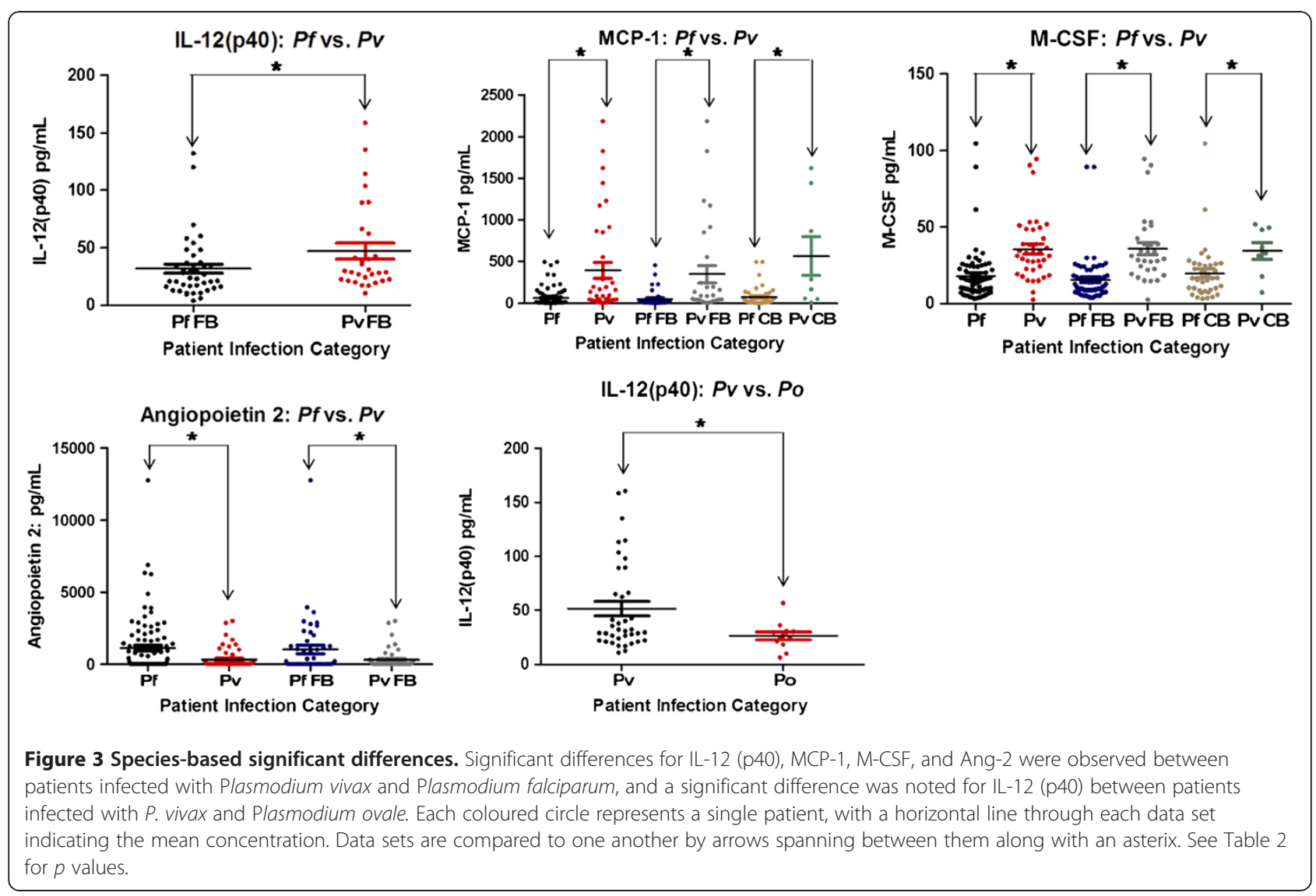


surmized to be a function of immune memory and protective factors for those who had been previously exposed. The data highlights that IL-12 (p40) and EGF are potential markers of disease severity. It was noted that IL-12 (p40) was significantly increased and EGF significantly decreased in $\mathrm{CB}$. If $\mathrm{CB}$ are more vulnerable to severe diseases as has been suspect clinically, then high IL-12 (p40) and low EGF may predict worse outcomes. Consistent with this observation, IL-12 (p40) is a pro-inflammatory cytokine produced by mononuclear phagocytes that activates T-helper 1 cells (Th1) and is important for assisting in innate immune responses against intracellular microbes [14]. However, some studies have shown elevated plasma levels of IL-12 (p40) attributed to mild cases of malaria, with low levels being associated with severe malaria [14,21]. Other studies counter these claims and indicate elevated levels have been seen in individuals with severe malaria in certain Asian populations $[13,22]$. Additionally, a pre-disposition to severe malaria has been shown to be caused by certain promoter variants that cause an increase in IL-12 production [22,23]. The findings provide support to the idea that IL-12 is more abundant in severe malaria. Further studies into the genetic properties of

Table 2 Statistical summary for comparisons made in this study

\begin{tabular}{|c|c|c|c|c|c|c|}
\hline Analysis & Marker & Data Set 1 & Mean Set 1 & Data Set 2 & Mean Set 2 & $P$ Value \\
\hline \multirow[t]{2}{*}{$\overline{C B}$ vs FB } & $\|-12(p 40)$ & $C B n=57$ & $70.39 \pm 9.95$ & $\mathrm{FB} n=87$ & $36.24 \pm 3.28$ & $<0.01$ \\
\hline & EGF & $C B n=37$ & $9.477 \pm 1.52$ & $\mathrm{FB} n=60$ & $14.82 \pm 1.68$ & 0.0315 \\
\hline$\overline{\text { Pf CB vs Pf FB }}$ & IL-12(p40) & $\mathrm{CB} n=46$ & $70.31 \pm 11.79$ & $F B n=42$ & $31.53 \pm 4.09$ & $<0.01$ \\
\hline \multirow[t]{2}{*}{ Pv Age: $55+$ vs 19-54 } & IL-6 & $55+n=10$ & $858.8 \pm 454.3$ & $19-54 n=21$ & $134.7 \pm 82.59$ & 0.0366 \\
\hline & MCSF & $55+n=10$ & $45.66 \pm 8.25$ & $19-54 n=23$ & $31.21 \pm 2.82$ & 0.0433 \\
\hline \multirow[t]{3}{*}{ Species: Pf vs Pv } & MCP-1 & Pf $n=86$ & $63.78 \pm 11.44$ & $P \vee n=38$ & $392.5 \pm 95.45$ & $<0.01$ \\
\hline & M-CSF & Pf $n=69$ & $17.61 \pm 2.05$ & $P \vee n=38$ & $35.6 \pm 3.38$ & $<0.01$ \\
\hline & Angiopoietin 2 & Pf $n=96$ & $1120 \pm 196.5$ & $P \vee n=59$ & $336.5 \pm 88.21$ & $<0.01$ \\
\hline Species: Pv vs Po & $\mathrm{IL}-12(\mathrm{p} 40)$ & $P \vee n=38$ & $51.4 \pm 6.76$ & Po $n=12$ & $26.4 \pm 3.73$ & 0.0478 \\
\hline \multirow[t]{2}{*}{ Species: Pf CB vs Pv CB } & MCP-1 & $\mathrm{Pf} C B \mathrm{n}=46$ & $74.74 \pm 16.63$ & $P \vee C B n=8$ & $563.4 \pm 233.2$ & $<0.01$ \\
\hline & MCSF & $\mathrm{Pf} C \mathrm{CB} \mathrm{n}=39$ & $19.59 \pm 2.89$ & $P \vee C B n=8$ & $34.37 \pm 5.59$ & 0.0367 \\
\hline \multirow[t]{4}{*}{ Species: Pf FB vs Pv FB } & MCP-1 & Pf FB $n=40$ & $51.18 \pm 15.43$ & $P \vee F B n=30$ & $347 \pm 104.3$ & $<0.01$ \\
\hline & M-CSF & $\mathrm{PfFB} n=30$ & $15.32 \pm 1.87$ & $P \vee F B n=30$ & $35.3 \pm 4.05$ & $<0.01$ \\
\hline & IL-12 (p40) & PfFB $n=42$ & $31.53 \pm 4.09$ & $P \vee F B n=30$ & $47 \pm 7.01$ & 0.0467 \\
\hline & Angiopoietin 2 & Pf FB $n=48$ & $1033 \pm 296.6$ & $P \vee F B n=49$ & $310.8 \pm 97.81$ & $<0.01$ \\
\hline \multirow[t]{2}{*}{ Travel: West Africa vs other African regions } & $\mathrm{IFN}-\gamma$ & West $n=27$ & $11.88 \pm 1.98$ & Other $n=12$ & $22.5 \pm 6.16$ & 0.0414 \\
\hline & IL-6 & West $n=22$ & $31.58 \pm 6.28$ & Other $n=12$ & $120.1 \pm 56.27$ & 0.0426 \\
\hline
\end{tabular}


the IL-12 (p40) promoter in Canadian populations may aid in determining the connections between this study and the findings of others.

EGF binds to the epidermal growth factor receptor (EGFR) where it plays a key role in regulating cellular differentiation, proliferation, and survival [24]. EGF-like domains on $P$. falciparum surface proteins have been studied in great detail for vaccine research $[16,24,25]$, but studies looking at the relationship between EGF levels circulating in the body and malaria infection have not been explored in any detail. The findings indicate a tendency for FB individuals to have a significantly higher level of circulating plasma EGF than CB individuals, but further research will need to be completed to determine the significance of this result and the potential relevance increased levels may have in protecting against severe disease.

Studies focusing on older adults [26], as well as Africanbased epidemiological data for children [27] show a tendency for these groups to be stricken with malaria more frequently than other age groups, with mortality often at the highest among young children. The results indicated that in $P$. vivax infections, adults over age 55 had more pronounced IL- 6 and M-CSF production. IL- 6 is a pro-inflammatory cytokine. It is produced by numerous cell types including monocytes, endothelial cells and fibroblasts [28] and has a variety of biological roles, including the stimulation of $\mathrm{B}$ and $\mathrm{T}$ cell differentiation [29]. IL-6 has been shown to be elevated in Mali children with severe $P$. falciparum malaria [30], in a study of severe cases of malaria among 40 adults aged 21-65 [28], and has been found elevated in patients with cerebral malaria and renal failure [31]. The findings corroborate these studies, suggesting further potential of IL-6 as a marker of disease severity. It was surprising to not find the same result in $P$. falciparum infections, where the role of IL-6 was expected to be more pronounced.

Macrophage colony-stimulating factor (M-CSF) enhances macrophage activity, and it has been suggested that when elevated in malaria, it may lead to an increase in macrophage-mediated platelet destruction [17]. Elevated levels of M-CSF have been attributed to severe cases of P. falciparum malaria, where platelet destruction and the release of P-selectin plays a role in thrombocytopaenia associated with severe disease [17]. M-CSF has also been noted to be elevated in P. vivax cases [17], so the results indicating a higher level of M-CSF in older patients with $P$. vivax infection appear reasonable. It was noted that when all patient data was divided by species of infection, $P$. vivax had a higher mean cytokine response for M-CSF than P. falciparum. This corresponds for the tendency of $P$. vivax to elicit a stronger immune response in patients [32], though this is not necessarily a marker of severity, but is a marker of interest. The significant difference was also seen between M-CSF of just FB patients with $P$. vivax compared to P. falciparum, as well as just $\mathrm{CB}$ patients.

Monocyte chemo-attractant protein 1 (MCP-1) is required for migration of monocytes and macrophages to sites of inflammation [33], and has been associated with several neuro-inflammatory, and other inflammatory diseases, including HIV-1 encephalitis, and rheumatoid arthritis [34,35]. MCP-1 studies previously showed it to have no association with severe and cerebral malaria in P. falciparum-infected patients from Thailand and India $[15,36]$. The results indicated a substantially higher level of MCP-1 in patients infected with P. vivax compared to $P$. falciparum within the full data set, and in comparisons of just FB individuals, and just $\mathrm{CB}$ individuals. This finding again corresponds to the tendency of $P$. vivax to have a greater inflammatory response than $P$. falciparum, as does the results with just FB blood samples where the $P$. vivax patients again had a higher level of IL-12 (p40) than P. falciparum patients. The increased levels of circulating IL-12 (p40) in P. vivax compared to $P$. ovale again attests to the substantial inflammatory response associated with $P$. vivax.

Infections obtained through travel to different areas of Africa also showed a significant difference in patient immune response. A substantial amount of the $P$. falciparum blood samples were from travellers who had been to Western Africa, with most having visited Ghana and Nigeria. All those individuals who had contracted malaria in Western African countries (which included Nigeria and Ghana), were grouped and compared against travellers who were known to have been in other regions of Africa. Significantly lower levels of IL-6 and IFN- $\gamma$ were found in the Western Africa subset. One study has indicated that there can be reductions in total cases of severe malaria with increasing transmission rates [37], possibly indicating increased transmission of malaria in Western Africa triggers a lower intensity immune response. An additional possibility could be the presence of unique strains in West Africa triggering a different immune response compared to other African strains.

Of the cytokines that were found to be associated with travel, Il- 6 has been shown to often be elevated in severe cases of malaria $[28,30,31]$ as mentioned previously. It was also observed to be a contributor to disease outcome in non-endemic areas of India compared to endemic areas [38]. Studies on children in Sudan, an area of unstable malaria transmission, indicate a tendency for severe cases of $P$. falciparum malaria to have elevated levels of IFN- $\gamma$ [39]; this is also observed in severe cases of $P$. falciparum malaria in India among patients aged five to 75 [13]. Studies on P. vivax-infected patients from the Brazilian Amazon indicate plasma levels of IFN- $\gamma$ increase linearly with a gradual augmentation of disease severity [40]. 


\section{Activation of endothelium as assessed by Ang-1 and -2 serum levels in malaria infections}

Ang-2 levels have been reported to be elevated in cases of complicated severe malaria compared to uncomplicated malaria [12,19]. Ang-2 primes vascular endothelial cells to exogenous cytokines, and at high concentrations can trigger vascular permeability $[41,42]$. The results indicate that in P. falciparum infections, a higher level of Ang-2 is present than in P. vivax infections. These findings appeared in a comparison of all the patient data, as well as in a comparison of just FB patients with $P$. falciparum compared to $\mathrm{FB}$ with $P$. vivax. P. falciparum is generally considered to be more virulent than other causes of malaria, and has been observed to more commonly lead to severe cases of malaria [43]. Higher levels of Ang-2 in $P$. falciparum infections are consistent with this endothelium activation factor as Ang-2 and P. falciparum are both associated with more severe disease instances. A previous study reported that Ang-2 was more strongly elevated in the peripheral blood of individuals with $P$. vivax malaria compared to individuals with $P$. falciparum infection [44], which corresponds to its tendency to trigger a stronger cytokine response in infected patients.

\section{Conclusion}

The significantly higher mean levels of IL-12 (p40) and significantly lower levels of EGF found in CB travellers may serve to be useful prognostic markers of disease severity and help guide clinical management upon their return. IL-6 and M-CSF in older adults, MCP-1, IL-12 (p40) and M-CSF in P. vivax-infected patients, and Ang2 in P. falciparum-infected patients may also prove useful in understanding age-associated and species-specific host immune responses. Regional differences in host immune response to malaria infection within the same species ( $P$. falciparum) may be related to unique strains circulating in West Africa.

\section{Additional file}

Additional file 1: All comparisons made between various subsets of the data look for significant differences. Those italicized in red were significant, all others were not significant. Blanks represent instances where insufficient data was present $(\sim 1-5$ data points available) to make relevant comparisons.

\section{Abbreviations}

CB: Canadian-born; FB: Foreign-born; Pf: Plasmodium falciparum;

Pv: Plasmodium vivax; Po: Plasmodium ovale; Pm: Plasmodium malariae.

\section{Competing interests}

The authors declare that they have no competing interests.

\section{Acknowledgements}

Funding for this study was provided in part from a Canadian Research Chair in Infectious Diseases and Inflammation (WCL) from the Canadian Institutes of Health Research (CIHR) and the Ontario Agency for Health Protection and Promotion (DRP).

\section{Author details}

${ }^{1}$ Public Health Ontario, Toronto, ON , Canada. ${ }^{2}$ Mount Sinai Hospital, Toronto, ON , Canada. ${ }^{3}$ Division of Infectious Diseases, Department of Medicine, University of Toronto, Toronto, ON , Canada. ${ }^{4}$ St Michael's Hospital, Toronto, ON , Canada. ${ }^{5}$ Sandra A Rotman Laboratory for Global Health, McLaughlinRotman Centre for Global Health, University Health Network, Toronto, ON Canada. ${ }^{6}$ Current address: Departments of Pathology \& Laboratory Medicine, Medicine, and Microbiology \& Infectious Diseases, The University of Calgary, Diagnostic \& Scientific Centre, Room 1W-416, 9-3535 Research Road NW, Calgary, AB T2L 2K8, Canada.

\section{Authors' contributions}

GM and RM performed the experiments, carried out the data analysis, and GM wrote the initial draft of the manuscript. RL assisted with sample preparation and helped to coordinate the study. JK preformed the cytokine multiplex assays. NR carried out the Angiopoietin ELISA assays. DRP, HZ, and WCL conceived the study, and participated in its design and coordination. All authors read and approved the final manuscript.

Received: 22 December 2011 Accepted: 3 May 2012

Published: 3 May 2012

\section{References}

1. Hay SI, Okiro EA, Gething PW, Patil AP, Tatem AJ, Guerra CA, Snow RW: Estimating the global clinical burden of Plasmodium falciparum malaria in 2007. PLoS Med 2010, 7:e1000290.

2. Tine JA, Lanar DE, Smith DM, Wellde BT, Schultheiss $P$, Ware LA, Kauffman EB, Wirtz RA, De Taisne C, Hui GS, Chang SP, Church P, Hollingdale MR, Kaslow DC, Hoffman S, Guito SP, Ballou WR, Sadoff JC, Paoletti E: NYVACPf7: a poxvirus-vectored, multiantigen, multistage vaccine candidate for Plasmodium falciparum malaria. Infect Immun 1996, 64:3833-3844.

3. Lovegrove JA, Hampton SM, Morgan JB, Marks V: Quantification of total IgG levels against alpha-casein in human serum samples. Biochem Soc Trans 1989, 17:1059-1060.

4. Leder K, Black J, O'Brien D, Greenwood Z, Kain KC, Schwartz E, Brown G, Torresi J: Malaria in travelers: a review of the GeoSentinel surveillance network. Clin Infect Dis 2004, 39:1104-1112.

5. Kain KC, Keystone JS: Malaria in travelers. Epidemiology, disease, and prevention. Infect Dis Clin North Am 1998, 12:267-284.

6. Toronto's Racial Diversity. [http://www.toronto.ca/toronto_facts/diversity.htm]

7. Entertainment and Tourism. [http://www.toronto.ca/toronto_facts/ entertainment tourism.htm].

8. Venkataraman R, Kellum JA: Defining acute renal failure: the RIFLE criteria. J Intensive Care Med 2007, 22:187-193.

9. Guidelines for the treatment of malaria 2nd edition. [http://www.who.int/ malaria/publications/atoz/9789241547925/en/index.html].

10. Kain KC, MacPherson DW, Kelton T, Keystone JS, Mendelson J, MacLean JD: Malaria deaths in visitors to Canada and in Canadian travellers: a case series. CMAJ 2001, 164:654-659.

11. Wahlgren M, Abrams JS, Fernandez V, Bejarano MT, Azuma M, Torii M, Aikawa M, Howard RJ: Adhesion of Plasmodium falciparum-infected erythrocytes to human cells and secretion of cytokines (IL-1-beta, IL-1RA, IL-6, IL-8, IL-10, TGF beta, TNF alpha, G-CSF, GM-CSF. Scand J Immunol $1995,42: 626-636$.

12. Yeo TW, Lampah DA, Gitawati R, Tjitra E, Kenangalem E, Piera K, Price RN, Duffull SB, Celermajer DS, Anstey NM: Angiopoietin-2 is associated with decreased endothelial nitric oxide and poor clinical outcome in severe falciparum malaria. Proc Natl Acad Sci U S A 2008, 105:17097-17102.

13. Prakash D, Fesel C, Jain R, Cazenave PA, Mishra GC, Pied S: Clusters of cytokines determine malaria severity in Plasmodium falciparum-infected patients from endemic areas of Central India. J Infect Dis 2006, 194:198-207.

14. Malaguarnera L, Imbesi RM, Pignatelli S, Simpore J, Malaguarnera M, Musumeci S: Increased levels of interleukin-12 in Plasmodium falciparum malaria: correlation with the severity of disease. Parasite Immunol 2002, 24:387-389

15. Jain V, Armah HB, Tongren JE, Ned RM, Wilson NO, Crawford S, Joel PK Singh MP, Nagpal AC, Dash AP, Udhayakumar V, Singh N, Stiles JK: Plasma 
IP-10, apoptotic and angiogenic factors associated with fatal cerebral malaria in India. Malar J 2008, 7:83.

16. Kaslow DC, Quakyi IA, Syin C, Raum MG, Keister DB, Coligan JE, McCutchan TF, Miller $\mathrm{LH}$ : A vaccine candidate from the sexual stage of human malaria that contains EGF-like domains. Nature 1988, 333:74-76.

17. Lee SH, Looareesuwan S, Chan J, Wilairatana P, Vanijanonta S, Chong SM, Chong $\mathrm{BH}$ : Plasma macrophage colony-stimulating factor and P-selectin levels in malaria-associated thrombocytopenia. Thromb Haemost 1997, 77:289-293.

18. Morrell CN, Srivastava K, Swaim A, Lee MT, Chen J, Nagineni C, Hooks JJ, Detrick B: Beta interferon suppresses the development of experimental cerebral malaria. Infect Immun 2011, 79:1750-1758.

19. Lovegrove FE, Tangpukdee N, Opoka RO, Lafferty El, Rajwans N, Hawkes M, Krudsood S, Looareesuwan S, John CC, Liles WC, Kain KC: Serum angiopoietin-1 and -2 levels discriminate cerebral malaria from uncomplicated malaria and predict clinical outcome in African children. PLoS One 2009, 4:e4912.

20. Inc. A: Procarta Cytokine Assay Kit User Manaual Specifically for Serum and Plasma Samples. 2009, vol. P/N 10483 Rev. 042509: Inc. A.

21. Luty AJ, Perkins DJ, Lell B, Schmidt-Ott R, Lehman LG, Luckner D, Greve B, Matousek P, Herbich K, Schmid D, Weinberg JB, Kremsner PG: Low interleukin-12 activity in severe Plasmodium falciparum malaria. Infect Immun 2000, 68:3909-3915.

22. Phawong C, Ouma C, Tangteerawatana P, Thongshoob J, Were T, Mahakunkijcharoen Y, Wattanasirichaigoon D, Perkins DJ, Khusmith S: Haplotypes of IL12B promoter polymorphisms condition susceptibility to severe malaria and functional changes in cytokine levels in Thai adults. Immunogenetics 2010, 62:345-356.

23. Marquet S, Doumbo O, Cabantous S, Poudiougou B, Argiro L, Safeukui I, Konate S, Sissoko S, Chevereau E, Traore A, Keita MM, Chevillard C, Abel L, Dessein AJ: A functional promoter variant in IL12B predisposes to cerebral malaria. Hum Mol Genet 2008, 17:2190-2195.

24. Herbst RS: Review of epidermal growth factor receptor biology. Int $J$ Radiat Oncol Biol Phys 2004, 59:21-26.

25. Zamani Z, Razavi MR, Sadeghi S, Naddaf S, Pourfallah F, Mirkhani F Arjmand M, Feizhaddad H, Rad ME, Tameemi M, Assmar M: Sequence diversity of the C-terminal region of Plasmodium falciparum merozoite surface protein 1 in southern Iran. Southeast Asian J Trop Med Public Health 2009, 40:1-9.

26. Casalino E, Le Bras J, Chaussin F, Fichelle A, Bouvet E: Predictive factors of malaria in travelers to areas where malaria is endemic. Arch Intern Med 2002, 162:1625-1630.

27. Newton CR, Taylor TE, Whitten RO: Pathophysiology of fatal falciparum malaria in African children. Am J Trop Med Hyg 1998, 58:673-683.

28. Kern P, Hemmer CJ, Van Damme J, Gruss HJ, Dietrich M: Elevated tumor necrosis factor alpha and interleukin- 6 serum levels as markers for complicated Plasmodium falciparum malaria. Am J Med 1989, 87:139-143.

29. Uyttenhove C, Coulie PG, Van Snick J: T cell growth and differentiation induced by interleukin-HP1/IL-6, the murine hybridoma/plasmacytoma growth factor. J Exp Med 1988, 167:1417-1427.

30. Lyke KE, Burges R, Cissoko Y, Sangare L, Dao M, Diarra I, Kone A, Harley R, Plowe CV, Doumbo OK, Sztein MB: Serum levels of the proinflammatory cytokines interleukin-1 beta (IL-1beta), IL-6, IL-8, IL-10, tumor necrosis factor alpha, and IL-12(p70) in Malian children with severe Plasmodium falciparum malaria and matched uncomplicated malaria or healthy controls. Infect Immun 2004, 72:5630-5637.

31. Angulo I, Fresno M: Cytokines in the pathogenesis of and protection against malaria. Clin Diagn Lab Immunol 2002, 9:1145-1152.

32. Anstey NM, Russell B, Yeo TW, Price RN: The pathophysiology of vivax malaria. Trends Parasitol 2009, 25:220-227.

33. Gu L, Tseng SC, Rollins BJ: Monocyte chemoattractant protein-1. Chem Immunol 1999, 72:7-29.

34. Cinque $P$, Vago $L$, Mengozzi M, Torri V, Ceresa D, Vicenzi E, Transidico $P$, Vagani A, Sozzani S, Mantovani A, Lazzarin A, Poli G: Elevated cerebrospinal fluid levels of monocyte chemotactic protein-1 correlate with HIV-1 encephalitis and local viral replication. AIDS 1998, 12:1327-1332.

35. Aguilar F, Gonzalez-Escribano MF, Sanchez-Roman J, Nunez-Roldan A: MCP-1 promoter polymorphism in Spanish patients with systemic lupus erythematosus. Tissue Antigens 2001, 58:335-338.

36. Dechkum N, Hananantachai H, Patarapotikul J, Ohashi J, Krudsood S, Looareesuwan S, Tokunaga K: Monocyte chemoattractant protein 1
(MCP-1) gene polymorphism is not associated with severe and cerebral malaria in Thailand. Jpn J Infect Dis 2006, 59:239-244.

37. Marsh K, Snow RW: Host-parasite interaction and morbidity in malaria endemic areas. Philos Trans R Soc Lond B Biol Sci 1997, 352:1385-1394.

38. Sinha S, Qidwai T, Kanchan K, Jha GN, Anand P, Pati SS, Mohanty S, Mishra SK, Tyagi PK, Sharma SK, Awasthi S, Venkatesh V, Habib S: Distinct cytokine profiles define clinical immune response to falciparum malaria in regions of high or low disease transmission. Eur Cytokine Netw 2010, 21:232-240.

39. Mirghani HA, Eltahir HG: TM AE, Mirghani YA, Elbashir MI, Adam I: Cytokine profiles in children with severe Plasmodium falciparum malaria in an area of unstable malaria transmission in central Sudan. J Trop Pediatr 2011, 57:392-395

40. Andrade BB, Reis-Filho A, Souza-Neto SM, Clarencio J, Camargo LM, Barral A, Barral-Netto M: Severe Plasmodium vivax malaria exhibits marked inflammatory imbalance. Malar J 2010, 9:13.

41. Thomas M, Augustin HG: The role of the Angiopoietins in vascular morphogenesis. Angiogenesis 2009, 12:125-137.

42. Fiedler U, Augustin HG: Angiopoietins: a link between angiogenesis and inflammation. Trends Immunol 2006, 27:552-558.

43. Greenberg $A E$, Lobel HO: Mortality from Plasmodium falciparum malaria in travelers from the United States, 1959 to 1987. Ann Intern Med 1990, 113:326-327.

44. Yeo TW, Lampah DA, Tjitra E, Piera K, Gitawati R, Kenangalem E, Price RN, Anstey NM: Greater endothelial activation, Weibel-Palade body release and host inflammatory response to Plasmodium vivax, compared with Plasmodium falciparum: a prospective study in Papua, Indonesia. $J$ Infect Dis 2010, 202:109-112.

doi:10.1186/1475-2875-11-148

Cite this article as: MacMullin et al:: Host immune response in returning travellers infected with malaria. Malaria Journal 2012 11:148.

\section{Submit your next manuscript to BioMed Central and take full advantage of:}

- Convenient online submission

- Thorough peer review

- No space constraints or color figure charges

- Immediate publication on acceptance

- Inclusion in PubMed, CAS, Scopus and Google Scholar

- Research which is freely available for redistribution 